\title{
Transverse Optical Mode Patterns for an RF Excited Ar-He-Xe Laser
}

\author{
Frans J. Blok, Nikolay N. Elkin, Igor V. Kochetov, Anatoly P. Napartovich, Peter J. M. Peters, \\ Sergei A. Starostin, Vera N. Troshchieva, Yuri B. Udalov, and W. J. Witteman
}

\begin{abstract}
Transverse optical modes for an RF excited Ar-He-Xe laser are studied both experimentally and theoretically. A diffraction model for a waveguide with a nonsaturable gain and refractive index gradients placed between two plane mirrors is formulated. The effects of gain and diffraction index gradients and of diffraction in free space are evaluated for typical experimental conditions. A direct comparison between theoretical mode patterns and experimentally measured ones at distances of 17 and $114 \mathrm{~cm}$ from the output mirror demonstrated a satisfactory agreement for various laser wavelengths and gas mixture compositions.
\end{abstract}

Index Terms-Gas lasers, laser beams, laser modes, modeling, optical resonators.

\section{INTRODUCTION}

$\mathbf{T}$ HE RECENT development of the RF-excited Ar-He-Xe laser has resulted in a substantial increase of the $\mathrm{CW}$ output power from relatively compact devices [1], [2] (watts compared to milliwatts of laser output power for dc excited systems). An output power of more than $5 \mathrm{~W}$ and an increase of the operational spectral range has been reported [3], [4]. This has opened new fields of applications for such a laser. One of the most promising applications seems to be the development of an eye-safe $2-\mu \mathrm{m}$ LIDAR based on this system.

In [5], it was shown that the small-signal gain profile for the $\mathrm{RF}$ excited $\mathrm{Ar}-\mathrm{He}-\mathrm{Xe}$ laser is strongly inhomogeneous in the direction transverse to the electrodes and has two maxima in the vicinity of the electrodes. This experimental fact can be explained from the point of view of general RF discharge properties. In an RF discharge, a high electric field gradient exists in the vicinity of the electrodes (see, for instance, [6]). Here high energy electrons are formed. It is well known that one of the necessary conditions for efficient operation of the atomic Xe laser is the presence of such high-energy electrons.

The low electron density in this discharge region is also favorable for the inversion of the laser levels because it is known that a high density of low-energy electrons (as in the case of the dc-excited Xe laser or RF bulk plasma)

Manuscript received March 16, 1999; revised August 16, 1999. This work was supported in part by the Dutch Scientific Foundation (NWO), Foundation for Technical Sciences (STW), and by the Fundamental Research of the Matter (FOM).

F. J. Blok, P. J. M. Peters, S. A. Starostin, Y. B. Udalov, and W. J. Witteman are with the Department of Applied Physics, University of Twente, 7500 AE Enschede, The Netherlands.

N. N. Elkin, I. V. Kochetov, A. P. Napartovich, and V. N. Troschchieva are with TRINITI, 142092, Troitsk, Moscow, Russia.

Publisher Item Identifier S 0018-9197(99)09430-0. leads to the electron collisional mixing of laser levels and subsequently decreases the inversion. The inhomogeneity of the RF discharge parameters thus leads to the inhomogeneity of the gain profile.

The excess energy from the excited region should be removed by heat conduction to the walls, resulting in gas temperature and refractive index gradients. The refractive index gradient may strongly influence the waveguide mode shape and losses and the laser beam quality, which is an important parameter for applications [7]. This effect was taken into account in a model for the Ar-He-Xe laser [8]. On the other hand, the mirrors of the resonator are placed as a rule at some distance from the waveguide ends. Therefore, the actual cavity mode is generally formed as a result of a combination of free-space diffraction, reflection from mirrors, and waveguide propagation.

Our purpose is to incorporate in the model correctly all of the above-mentioned factors and to compare the predicted mode patterns with the experimentally measured ones. Recently, the transverse mode properties of an unstable cavity $\mathrm{He}-\mathrm{Xe}$ laser have been described [9].

\section{EXPERIMENT}

The spatial structure of the $\mathrm{Ar}-\mathrm{He}-\mathrm{Xe}$ laser output was studied with the experimental setup described below (see Fig. 1). A capacitively coupled transverse RF gas discharge with an excitation frequency of $109 \mathrm{MHz}$ was used for laser pumping. An RF amplifier $\{1\}$ with a maximum output power of $1 \mathrm{~kW}$ was connected through a $\pi$-type matching circuit $\{2\}$ to the laser head $\{3\}$. The discharge inside the laser head was confined within a slab waveguide formed by two water-cooled electrodes each $15 \mathrm{~mm}$ wide and $370 \mathrm{~mm}$ long. The interelectrode distance was $2 \mathrm{~mm}$. Dielectric side-walls were used. The resonator consisted of two flat mirrors: a goldcoated total reflecting mirror $\{4\}$ and an outcoupling mirror $\{5\}$ with $40 \%$ reflectivity. The distance between mirrors was $390 \mathrm{~mm}$, while the distance from each mirror to the end of the waveguide was $10 \mathrm{~mm}$. For the intensity profile registration, a SPIRICON array $\{6\}$ (model LP 128-22- $\mathrm{BaF}_{2}$ ) together with a beam chopper $\{7\}$ were used. The array consisted of 128 pyroelectric elements, each $0.2 \mathrm{~mm}$ in size. The distance between the output mirror and the array was varied along the optical axis from 17 to $114 \mathrm{~cm}$ without realigning. To control the laser output power, a broad-band power/energy meter $\{8\}$ (Melles-Griot) was used. It was possible to measure the output power for each particular line present in the laser oscillation 


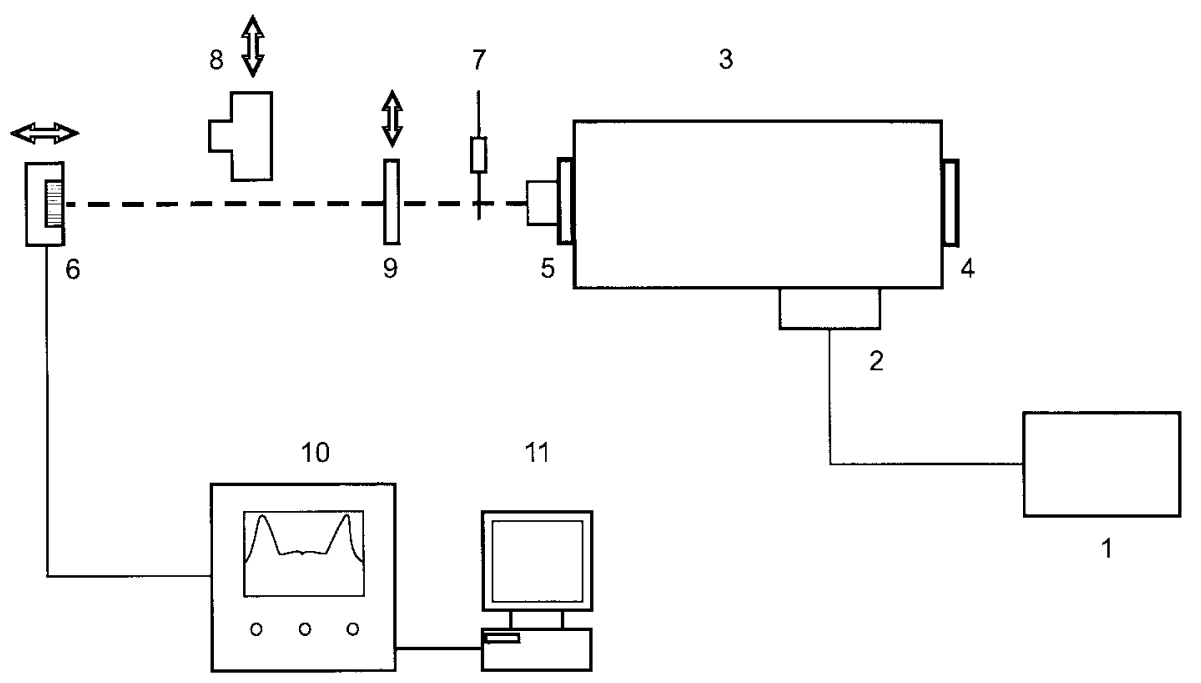

Fig. 1. Experimental setup. 1. RF. amplifier. 2. $\pi$-type matching circuit. 3. Laser head. 4. A gold-coated reflecting mirror. 5. An outcoupling mirror with 40\% reflectivity. 6. A SPIRICON array. 7. A beam chopper. 8. A broad-band power/energy meter. 9. Narrow-band spectral filters. 10. A digital oscilloscope. 11. PC.

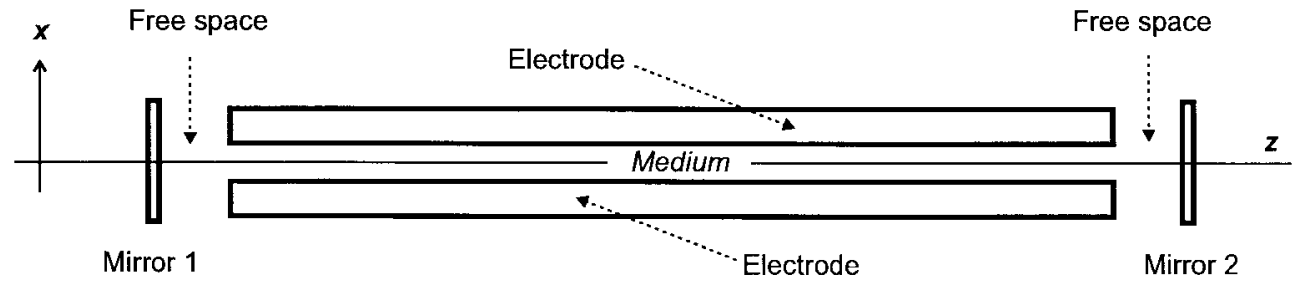

Fig. 2. Resonator configuration.

spectrum. To select the desired laser line narrow band spectral filters $\{9\}$ were used. These filters were also used in the laser beam profile measurements. The signal from the array was registered with a digital oscilloscope $\{10\}$ and stored in the memory of a PC $\{11\}$. Homemade data acquisition software written in LabView was used for data acquisition and processing.

The measurements were done for two laser gas mixtures. The optimized gas mixture (Ar: $\mathrm{He}: \mathrm{Xe}=59.5: 40: 0.5$, mixture 1) produced the highest laser output of $1.7 \mathrm{~W}$ on the $2.03-\mathrm{mm}$ and $2.65-\mu \mathrm{m}$ laser lines. To evaluate experimentally the effect of the refractive index gradient on the output intensity profiles within the waveguide, a helium rich mixture (Ar:He:Xe $=9.5: 90: 0.5$, mixture 2) with much smaller index variations was also studied. This mixture delivered a maximum laser output of $0.3 \mathrm{~W}$ with the 2.03-, 2.65-, and $3.51-\mu \mathrm{m}$ lines present in the laser output spectrum.

In both cases, the gas pressure was 150 mbar and the input power was $350 \mathrm{~W}$. It should be mentioned that the observed structure of the output laser beam was almost insensitive to the RF input power even when the laser power changed substantially. The spectral resolution of the bandpass filters was not high enough to distinguish between the 2.63- and 2.65$\mu \mathrm{m}$ lines; however, it is known from our previous experiments that the $2.65-\mu \mathrm{m}$ line dominates [10].

\section{NUMERICAL METHODOLOGY}

A two-dimensional (2-D) numerical code for the slab geometry was developed. The propagation of the field was in the $z$ direction. In the $x$ direction, a narrow spacing $(2 \mathrm{~mm}$ in our experiments) was assumed. The mode pattern in the lateral direction ( $15 \mathrm{~mm}$ in our experiments) was assumed to be fixed.

The intracavity radiation field is described by a complex scalar field of the form

$$
\begin{aligned}
E(x, z, t)=\left(E_{+}(x, z) \exp (i k z)+E_{-}(x, z)\right. \\
\cdot \exp (-i k z)) \exp (-i \omega t) \exp (-\delta t)
\end{aligned}
$$

where $\omega=k c$ is the frequency and $\delta$ is the decay rate.

In the free space regions, the slowly varying envelopes satisfy the parabolic equation

$$
\pm 2 i k \frac{\partial E_{ \pm}}{\partial z}+\frac{\partial^{2} E_{ \pm}}{\partial x^{2}}-i k g_{t} E_{ \pm}=0
$$

where $g_{t}=2 \delta / \mathrm{c}$.

The schematics diagram of the resonator containing a waveguide with an active medium, two mirrors, and two free-space regions is shown in Fig. 2.

The propagation of the field in the resonator was described by a round-trip propagation operator $\mathbf{P}$. Operator $\mathbf{P}$ was a combination of several operators each calculated separately: $\mathbf{P}_{1}$ describes the reflection from mirror $2, \mathbf{P}_{\mathbf{2}}$ is the freespace propagation from mirror 2 to the waveguide, $\mathbf{P}_{\mathbf{3}}$ is the propagation in the waveguide, $\mathbf{P}_{\mathbf{4}}$ is the free-space propagation from the waveguide to mirror $1, \mathbf{P}_{\mathbf{5}}$ is the reflection from mirror $1, \mathbf{P}_{\mathbf{6}}$ is the free-space propagation to the waveguide, $\mathbf{P}_{\mathbf{7}}$ is the propagation in the waveguide, and $\mathbf{P}_{\mathbf{8}}$ describes the free-space propagation from the waveguide to mirror 2 . 
On mirrors 1 and 2, the electric field satisfies the boundary conditions

$$
E_{+}(x, 0)=-E_{-}(x, 0)
$$

and

$$
E_{-}(x, L)=-E_{+}(x, L) \exp (i \phi) \rho_{2}
$$

where $\varphi=2 k L(\bmod 2 \pi)$ and $z=0$ is the position of mirror 1 (assumed to be totally reflecting), the second mirror 2 is positioned at $z=L$, and $\rho_{2}$ is the reflectance of the output mirror 2.

The coupling of the radiation from free space to the waveguide was calculated numerically by projection of the input wave field into the eigenmodes of the waveguide.

The propagation of the field within the waveguide was calculated using a split-operator technique taking spatial steps separately for the empty waveguide propagation and refraction-gain processes. The total length of the waveguide was divided into $N_{z}$ equal spatial layers, each with a thickness $h$. In our case, $N_{z}=40$.

The empty-waveguide propagation from $z$ to $z+h$ was calculated in three steps. First, a waveguide-mode expansion of the input field was made

$$
u(z, x)=\sum_{m} k_{m}(z) u_{m}(x)
$$

where $u(z, x)$ is the amplitude of the forward or backward wave and $u_{m}(x)$ is the empty-waveguide mode. The function $u_{m}(x)$ may be approximated with good accuracy by metallic waveguide functions (sine-functions).

Then the expansion coefficients in the output plane were calculated

$$
k_{m}(z+h)=k_{m}(z) \exp \left(i \beta_{m} h\right)
$$

where $\beta_{m}$ is the propagation constant of the $m$ th mode. The propagation constants were calculated from [11]

$$
\beta_{m}=-\frac{\kappa_{m}^{2}}{2 k}+i \chi_{m}
$$

with

$$
\chi_{m}=\frac{\kappa_{m}^{2}}{k(k a) \sqrt{n_{2}^{2}-1}}
$$

for TE modes and

$$
\frac{\kappa_{m}^{2} n_{2}^{2}}{k(k a) \sqrt{n_{2}^{2}-1}}
$$

for TM modes and $\kappa_{m}=\pi m / 2 a$ ( $a$ is the half-width of the waveguide). The index of the wall material is $n_{2}$.

In our case, the decay coefficients $\chi_{m}$ are very small. For a typical case, $\chi_{1} L_{m} \sim 10^{-4}$ where $L_{m}$ is the length of the waveguide.

Subsequently, the field $u(z+h, x)$ was calculated using (4). The refraction and gain processes in each spatial step were introduced by

$$
u(z+h, x)=u(z, x) \exp (i k(n(x)-1) h) \exp (g(x) h / 2)
$$

where $n(x)$ is the refractive index and $g(x)$ is the gain coefficient of the active medium.

In our calculation, $n(x)$ and $g(x)$ are taken as independent of the laser radiation intensity. The problem is formulated as a linear eigenproblem for the field on the second mirror $v(x)=E_{+}(x, L)$

$$
\mathbf{P} v=\gamma v
$$

with

$$
\gamma=\exp \left(-G_{t}-i \varphi\right)
$$

and

$$
G_{t}=\delta(2 L / c)
$$

where $\mathbf{P}$ is the round-trip propagation operator.

All eigenvalues, the decay rate $\delta$, and phase shift for a round trip $\varphi=2 k L(\bmod 2 \pi)$ are taken from (2)-(7). The composition of all these eigenvalues yield the complex eigenvalue $\gamma$. The eigenproblem (8) was solved numerically by a method developed by Krylov (its description can be found in [12], for example). The free-space propagation was calculated using a complex fast Fourier transform (FFT) algorithm. The modal expansion (4) in the waveguide was evaluated using a sine-FFT algorithm. One hundred twenty-eight modes were involved in the expansion.

To compare the theoretical results with the experimental data measured at distances of 17 and $114 \mathrm{~cm}$ from the outcoupling mirror, the mode intensity distribution was calculated in planes positioned at distances of 17 and $114 \mathrm{~cm}$ from the output mirror.

Therefore, the experimental values of the refractive index and gain distribution were put into the numerical model. The refractive index profile can be calculated easily provided that the electric discharge power density is known as a function of the transverse coordinate. For simplicity, it was assumed that the discharge power density was constant. Then, the refractive index can be easily calculated knowing the electrode temperature and gas pressure and composition. Evidently, there is a minimum at the center of the discharge and a maximum at the walls.

The gain profile in the calculations was found to be symmetric and resembled the measured small-signal gain distribution [5] (see Fig. 3). Two sets of experimental points are shown for conditions close to those realized in the present experiments. The observed scatter in the data illustrates the fluctuations in our experimental measurements. In theory, the gain profile was taken as a symmetric one because there is no reason for any asymmetry in the experiments, since the measurements of the gain and mode pattern were done with different devices.

\section{RESUlts OF THE Numerical SimUlations}

\section{A. Infinite Ideal Resonator}

As was mentioned above, the resulting cavity mode pattern depends on the inhomogeneity of the refractive index and laser gain, on the diffraction in free space, and on the coupling into the waveguide. To illustrate the role of each of these factors, let us present the calculated evolution of the simplest cavity 


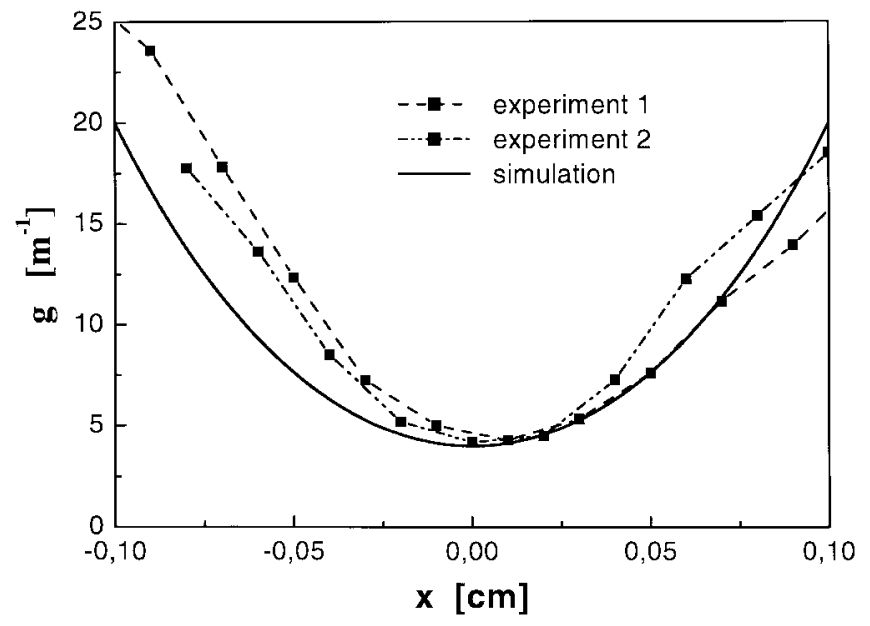

Fig. 3. Gain profiles: experimental points and numerical approximation.

modes starting from an empty waveguide with an infinite length. All calculations were performed for $\lambda=2.03 \mu \mathrm{m}$. In Fig. 4, the near-field mode intensity profiles are shown in columns 1 and 3 and the far-field distributions in columns 2 and 4 . The angle in the far field is scaled by $\lambda / a$. The lowest loss even-mode patterns are displayed in columns 1 and 2 and for the odd mode in columns 3 and 4 . The modulus of the eigenvalue $|\gamma|$ characterizing the cavity losses is shown as a caption in the picture of each mode for the near-field pattern. Note that the cavity round-trip loss, assuming ideal reflectors, is equal to $\left(1-|\gamma|^{2}\right)$.

The first three rows in Fig. 4 show the field distributions for infinite waveguides (an equivalent configuration is a cavity with mirrors placed close to the waveguide end facets). The results for an ideal or empty waveguide are shown in row 1; row 2 shows the results for a waveguide filled with a laser mixture with a high $\mathrm{He}$ content $(90 \%)$. In row 3, the laser mixture contains $40 \% \mathrm{He}$ and $60 \% \mathrm{Ar}$. The gases are heated in the discharge resulting in a close-to-parabolic refractive index profile. Due to the low refractive index increment $\Delta n=(n-1)$ for $\mathrm{He}$ and the higher thermal conductivity of the gas mixture with $90 \% \mathrm{He}$, the effect of mode distortion by refraction is much stronger for the $40 \% \mathrm{He}$ mixture, as can be seen in row 3. It is seen that the near-field mode pattern distortion by the nonuniform refraction is quite strong (this effect was evaluated earlier in [8]). It is worth noting that, in row 3, the near-field intensity profiles and the eigennumbers are practically the same for the even and odd modes. This is explained by the formation of two local waveguides between the walls and low refractive index region in the center. Each of these local waveguides has a set of localized waveguide modes. In fact, these waveguides are coupled by the evanescent waves. Estimates show that, for the mixture with $40 \% \mathrm{He}$, this coupling is very weak. It means that any small perturbation may disturb the coherence between the two beams and result in independent lasing in each local waveguide. The far-field distribution plots also show a very strong distortion of the waveguide mode pattern for the $40 \% \mathrm{He}$ mixture. In an infinite waveguide, losses are due only to the leakage of the waves into the waveguide walls. One can see from the first three rows that these losses grow with increasing refraction gradient; however, their magnitude remains very low.

\section{B. Resonator with Empty Space}

A further evolution of the mode intensity patterns in rows $4(90 \% \mathrm{He})$ and $5(40 \% \mathrm{He})$ shows the influence of the free spaces between the waveguide ends and the mirrors (see Fig. 2). The refractive index profiles were kept the same as for the infinite waveguide. Generally, this influence may be thought of as some mixing of waveguide modes owing to diffraction in free space. In the near field, this results in the formation of ripples in the intensity distribution. However, for the odd mode, the amplitudes of these ripples are comparable with the main peaks, and, as a result, the far-field distribution for this mode for the $40 \% \mathrm{He}$ mixture becomes much broader than for the infinite waveguide. Note that losses associated with waveguide coupling also grow with the increase of the refraction index, approaching 5\% for the odd mode.

The figures in the last row in Fig. 4 correspond to the same conditions as in row 5 (i.e., the $40 \%$ He mixture was considered), but now a nonuniform nonsaturable laser gain is added. The gain profile used was similar to the one shown in Fig. 3 with the maximum at the wall $g_{0}=10 \mathrm{~m}^{-1}$. However, the ratio of the maximum to minimum value was set equal to 20. It is interesting to note that the addition of gain leads to some smoothing of the mode patterns, with the odd modes becoming similar to the modes of the infinite waveguide (compare with row 3 on the right side). In a cavity with an active medium, the magnitude of the eigenvalue reflects both the cavity losses and gain. Therefore, in row 6 , it is scaled by a maximum magnitude possessed by the even mode.

Fig. 5 shows five modes patterns $(\lambda=2.03 \mu \mathrm{m})$ at the output mirror with the reflectance 0.36 calculated assuming the gain profile presented in Fig. 3 but scaled to a maximum value of $10 \mathrm{~m}^{-1}$. Mode patterns are presented for the position directly behind the output mirror and at distances for which experimental data are available, namely at 17 and $114 \mathrm{~cm}$ from the output mirror. A $40 \% \mathrm{He}$ mixture with a pressure of 150 mbar with an input power of $350 \mathrm{~W}$ was modeled. The modes are ordered by the magnitude of the eigenvalue. In Table I are presented the eigenfrequencies of the transverse modes in megahertz, modal gain $g_{n}$, waveguide coupling loss $\delta_{c}$, and waveguide wall absorption $\delta_{w}$. The following relationship between these quantities holds:

$$
\exp \left(2 g_{n} L\right) r\left(1-\delta_{n}^{t}\right)=\left|\gamma_{n}\right|^{2}
$$

where $\delta_{n}^{t}=\delta_{c}+\delta_{w}$ is the modal loss for waveguide coupling and leakage into the walls, $r$ is the output mirror reflectance, and $L=37 \mathrm{~cm}$ is the waveguide length. The width of the mode intensity distributions was calculated to be limited to 4 $\mathrm{mm}$ at $17 \mathrm{~cm}$ and to $16 \mathrm{~mm}$ at $114 \mathrm{~cm}$. The percentage of the laser beam flux falling within these intervals is shown in the figure captions of the second and third row. Except for the far-field $(114 \mathrm{~cm})$ pattern for mode \#3, the fraction of the flux falling within the selected intervals is high enough to provide 

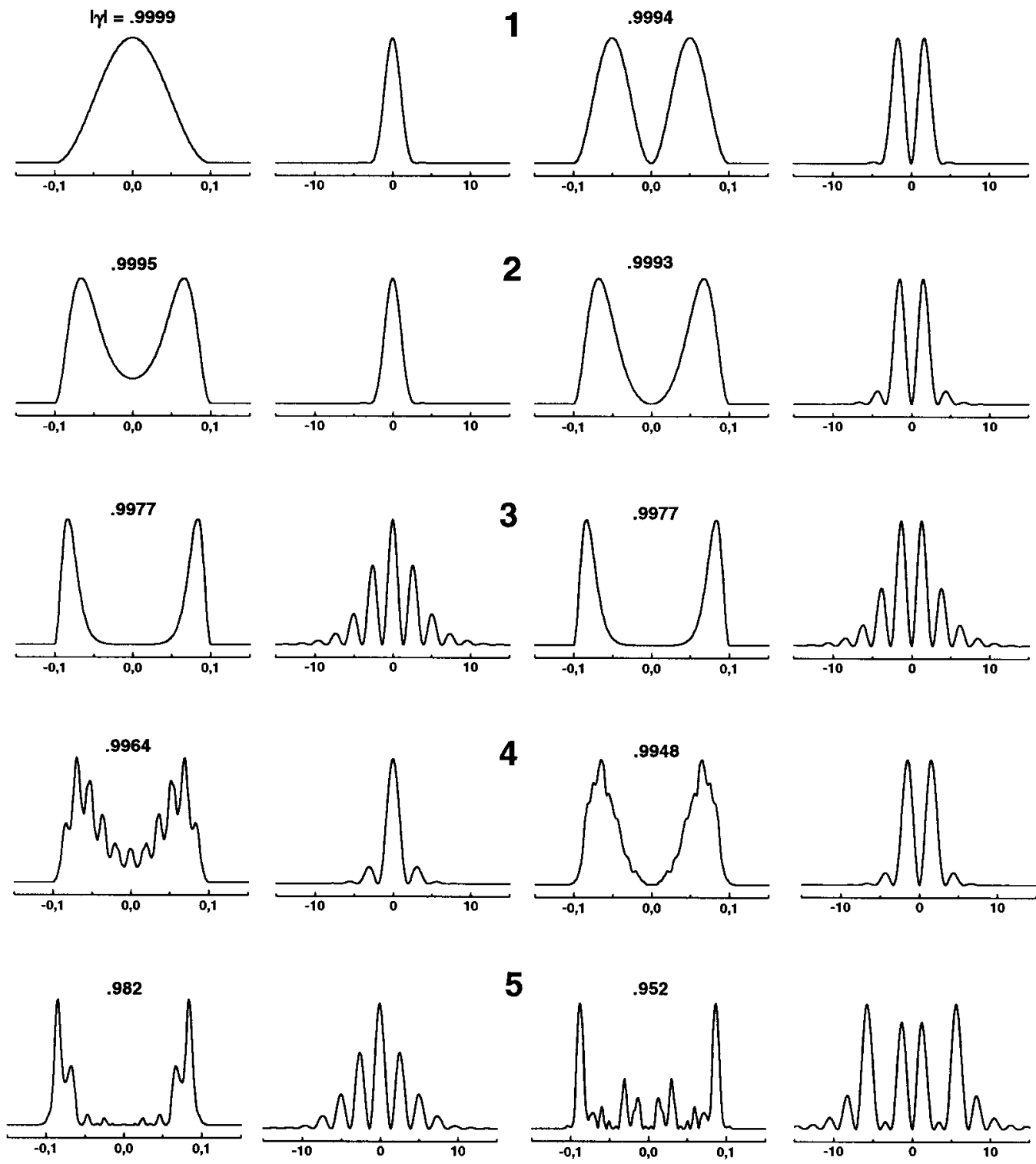
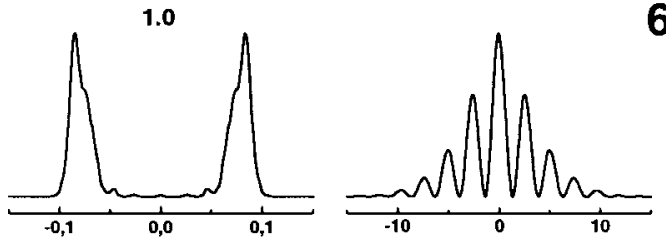

$x \quad[\mathbf{c m}]$

(a)

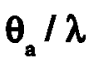

(b)

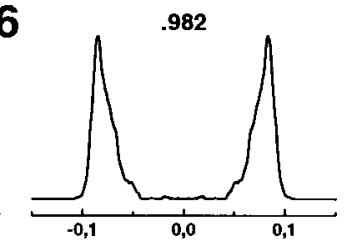

$x \quad[\mathbf{c m}]$

(c)

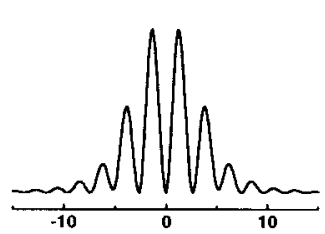

$\theta_{\mathrm{a}} / \lambda$

(d)

Fig. 4. Evolution of the near-field (column 1,3) and far-field (column 2,4) lowest loss mode patterns for different cavity conditions $(\lambda=2.03 \mu \mathrm{m}$ ). (a) Far field (even). (b) Far field (even). (c) Near field (odd). (d) Far field (odd).

meaningful results. It is seen from Table I that the first two modes are strongly discriminated against all others because the modal gain is higher, and the losses $\delta_{c}$ and $\delta_{w}$ are much smaller for these two modes. This means that only two modes (the first is odd and the second even) have the possibility to participate in lasing and to compete for gain. The most important factor is a good overlap of the mode intensity and gain profile.

\section{The Effects of Gain and Refraction Index Inhomogeneity}

Our model allows us to calculate the evolution of the field distribution along the propagation axis. Diffraction in free space is the reason for the difference between the waveguide modes and cavity modes. A cavity mode within the waveguide can be represented by a series expansion over the waveguide modes. During propagation, the phase differences between the 

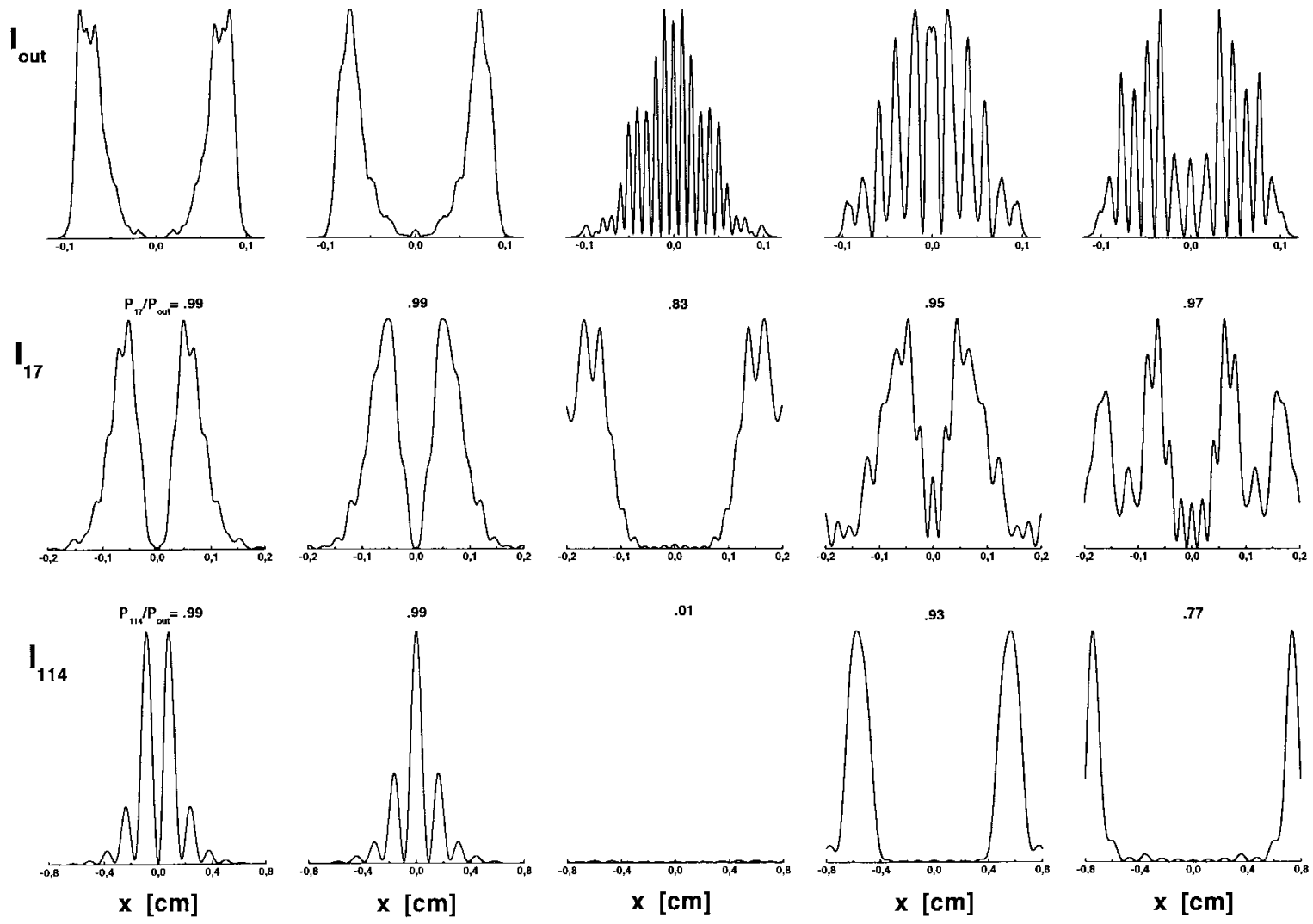

Fig. 5. Five mode patterns calculated at the output mirror plane $\left(I_{\text {out }}\right)$ and at distances of $17\left(I_{17}\right)$ and $114 \mathrm{~cm}\left(I_{114}\right)$ behind the output mirror $(\mathrm{Xe}: \mathrm{Ar}: \mathrm{He}=0.5: 59.5: 40 ; \lambda=2.03 \mu \mathrm{m})$.

TABLE I

EIGENFREQUENCIES OF Five TRANSVERSE MODES with CORRESPONDING Modal Gain $g_{m}$, WaVeguide Coupling Losses $\delta_{c}$, AND WaVeguide Wall Absorption $\delta_{w}$. Gas Mixture at 150-mbar Pressure (40\% He Content), an INPUt Power of $350 \mathrm{~W}$, AND LASER WaVELENGTH $\lambda=2.03 \mu \mathrm{m}$

\begin{tabular}{c|c|c|c|c|c}
\hline $\mathrm{n}$ & 1 & 2 & 3 & 4 & 5 \\
\hline$\Omega, \mathrm{MHz}$ & -252 & -249 & -33 & -162 & 330 \\
\hline $\mathrm{g}_{\mathrm{n}}, \mathrm{m}^{-1}$ & 5.75 & 5.69 & 4.57 & 4.47 & 4.46 \\
\hline$\delta_{\mathrm{c}, \%}$ & 1.15 & 1.34 & 3.58 & 3.98 & 7.34 \\
\hline$\delta_{\mathrm{w}}, \%$ & 0.44 & 0.81 & 11.4 & 4.14 & 5.33 \\
\hline
\end{tabular}

waveguide modes grow proportionally due to their respective differences between propagation constants. Therefore, one may expect that the cavity-mode intensity is not constant along the $z$ axis. This effect is illustrated in Fig. 6 for five even cavity modes calculated for a configuration where a waveguide with a length of $37 \mathrm{~cm}$ is placed close to the first mirror while the second mirror is placed at $2 \mathrm{~cm}$ from the other waveguide end. For these calculations performed for $\lambda=2.65 \mu \mathrm{m}$, a laser gas mixture with $90 \% \mathrm{He}$ was assumed. Further, a resonator with no gain and mirror losses was considered. In the upper row, the mode intensity is shown at the position of the first mirror attached to the waveguide end. The second row shows the mode intensity at the waveguide exit on the other side. The third row shows the wave phase distribution at the same waveguide exit. As one compares the intensity profiles in the upper and middle rows, a strong variation is seen. This demonstrates that the waveguide modes can not serve as a satisfactory approximation to the established cavity modes. In general, it is known that the phase of the wave field for the waveguide modes is a step-wise function with step differentials equal to $\pi$. In contrast, row 3 shows that the cavity mode at the waveguide exit has a strongly curved wave front.

Earlier papers about a cavity with an internal mirror placed inside the waveguide laser have been published [13]-[15]. An important experimental parameter is the distance from the waveguide end to the output mirror. Variation of this length usually caused a nonmonotonic change of the lowest cavity mode losses at a fixed radiation wavelength. This effect is explained by the sensitivity of the loss to the phase differences of waveguide modes gained during the propagation from the waveguide to the mirror and back. As a result, the patterns of the lowest loss modes may experience abrupt changes with variation of the distance (loss-curves-crossing phenomenon).

In this paper we introduce two new factors: the nonuniform gain and index of refraction. In Fig. 5, it is shown that all higher order modes are strongly discriminated against in favor of two modes by the spatial gain-loss distribution. Numerical simulations performed for the same conditions as in Fig. 5 for $\lambda=2.03 \mu \mathrm{m}$ showed that the effect of discrimination of the first two modes against all others is true for free-space distances to the output mirror in the range 1-22 $\mathrm{cm}$. The resulting dependencies of the maximum eigenvalue magnitude, modal gain coefficient, and round-trip loss for this mode with the maximum eigenvalue on the distance to the mirror are displayed in Fig. 7. It is seen that the maximum eigenvalue magnitude is a nonmonotonic function 

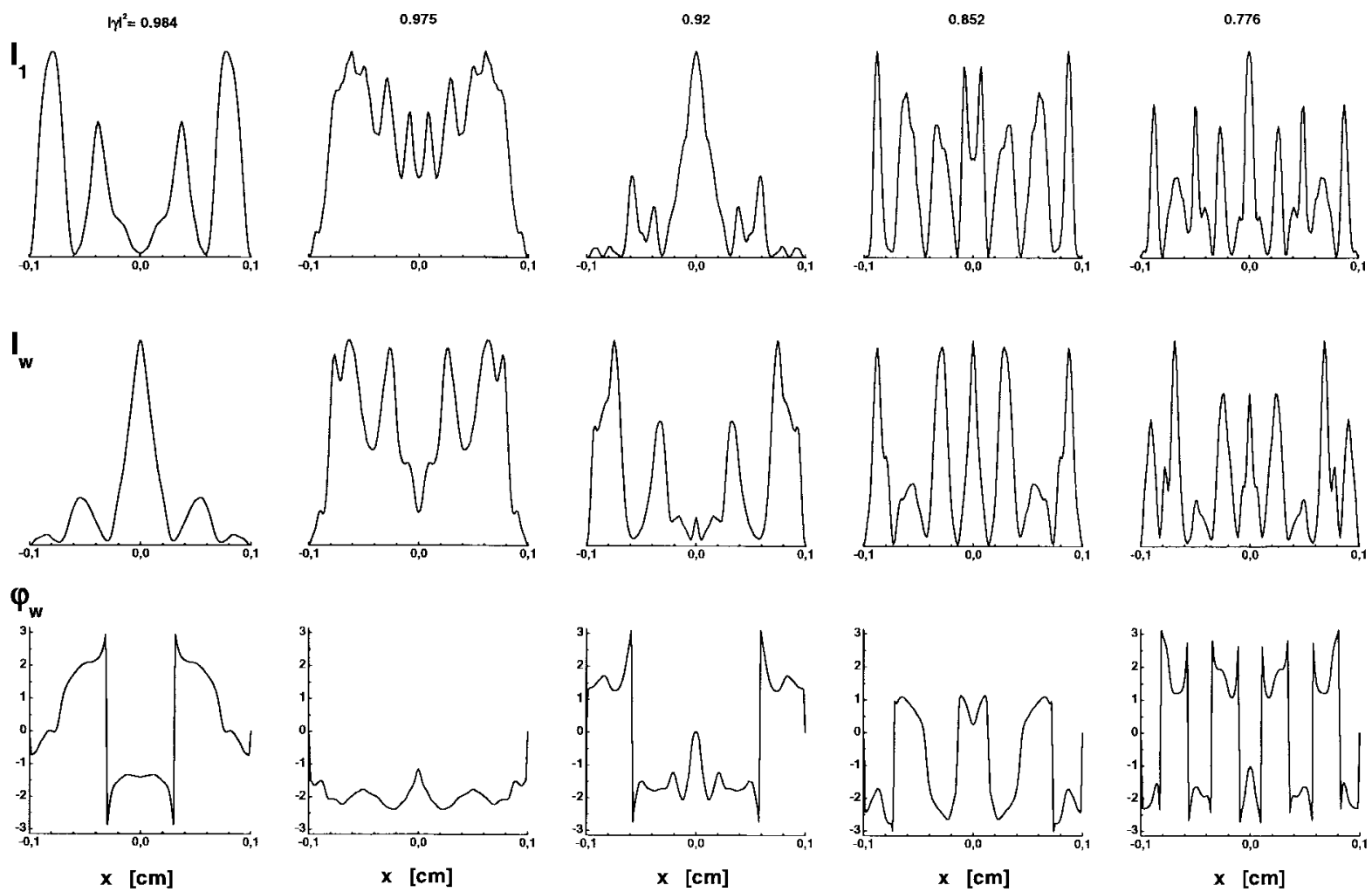

Fig. 6. Intensity profiles of the propagating modes at the waveguide end close to the first mirror $\left(I_{1}\right)$ and at the waveguide exit $\left(I_{w}\right)$. The modal phase distribution over the waveguide exit aperture is shown at the bottom row (Xe: $\mathrm{Ar}: \mathrm{He}=0.5: 9.5: 90 ; \lambda=2.65 \mu \mathrm{m})$.

of the distance, and, for a distance around 12 and $15 \mathrm{~cm}$, a mode change took place. The even mode became the dominant one only in the interval between 12 and $14.7 \mathrm{~cm}$, which means that, by an appropriate choice of the distance between the waveguide and the output mirror, single-mode lasing is anticipated. Comparing the behavior of the modal gain and loss around the points of mode hopping, one can conclude that the variation of the gain plays a more important role than that of the diffraction losses. This is explained by comparatively high values of the gain coefficient used in our calculations. In other words, the symmetry of the dominant mode depends on the shape of the gain and the refractive index profiles and the magnitude of the small-signal gain, as well. It is also interesting that, while the magnitude of the eigenvalue is a continuous function of the distance, the modal gain and loss experience a discontinuous change in the moments of mode hopping.

To illustrate the role played by the laser wavelength, Fig. 8 shows the calculated results for exactly the same conditions as in Fig. 5 except that the wavelength was set at $2.65 \mu \mathrm{m}$. Table II presents the mode frequency, modal gain, waveguide coupling, and wall absorption losses. One can see that the odd mode has the highest gain. The discrimination between the two first modes and all higher order modes is (similar to $\lambda=2.03 \mu \mathrm{m}$ ) sufficiently strong. It is seen that modes \#4 and \#5 have an anomalously high divergence. However, the difference between the mode patterns of the practically important first two modes for $\lambda=2.03 \mu \mathrm{m}$ and $\lambda=2.65 \mu \mathrm{m}$ is not as great.

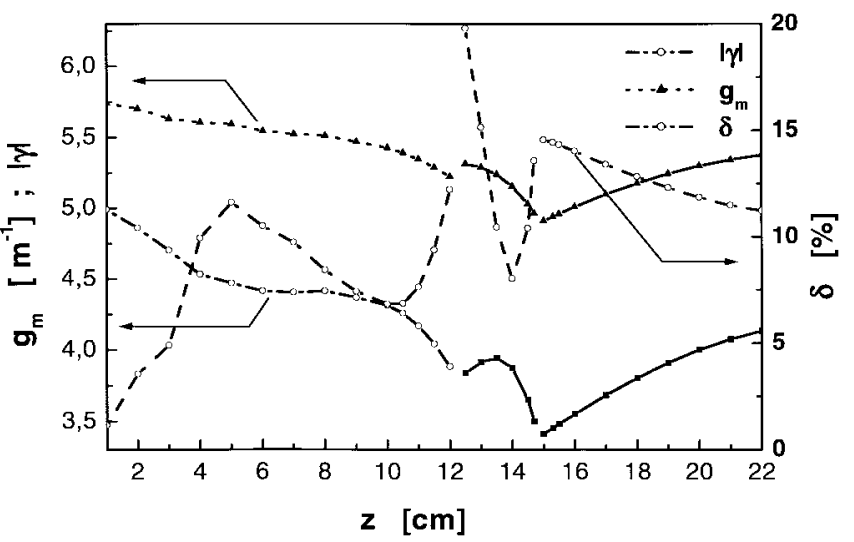

Fig. 7. Modal gain coefficient and loss per round trip as functions of the free-space distance from the waveguide to the output mirror. All other conditions are kept the same as those in Fig. 5. A mode change occurs where the curves are not connected.

Special studies were performed on how the gain amplitude influences the mode pattern. Different gain profiles and gas mixtures at a gas pressure of 150 mbar were considered with refractive index gradients calculated for an input power of $350 \mathrm{~W}$. It was shown numerically that the modal gain $g_{m}$ depends linearly on the maximum value of the gain coefficient $g_{0}$, provided all other conditions are the same. It means that

$$
g_{n}=\alpha_{n} g_{0}
$$

where $\alpha_{n}$ is a factor of proportionality. It was shown by direct simulations that this relationship holds for $g_{0} \leq 10 \mathrm{~m}^{-1}$. 

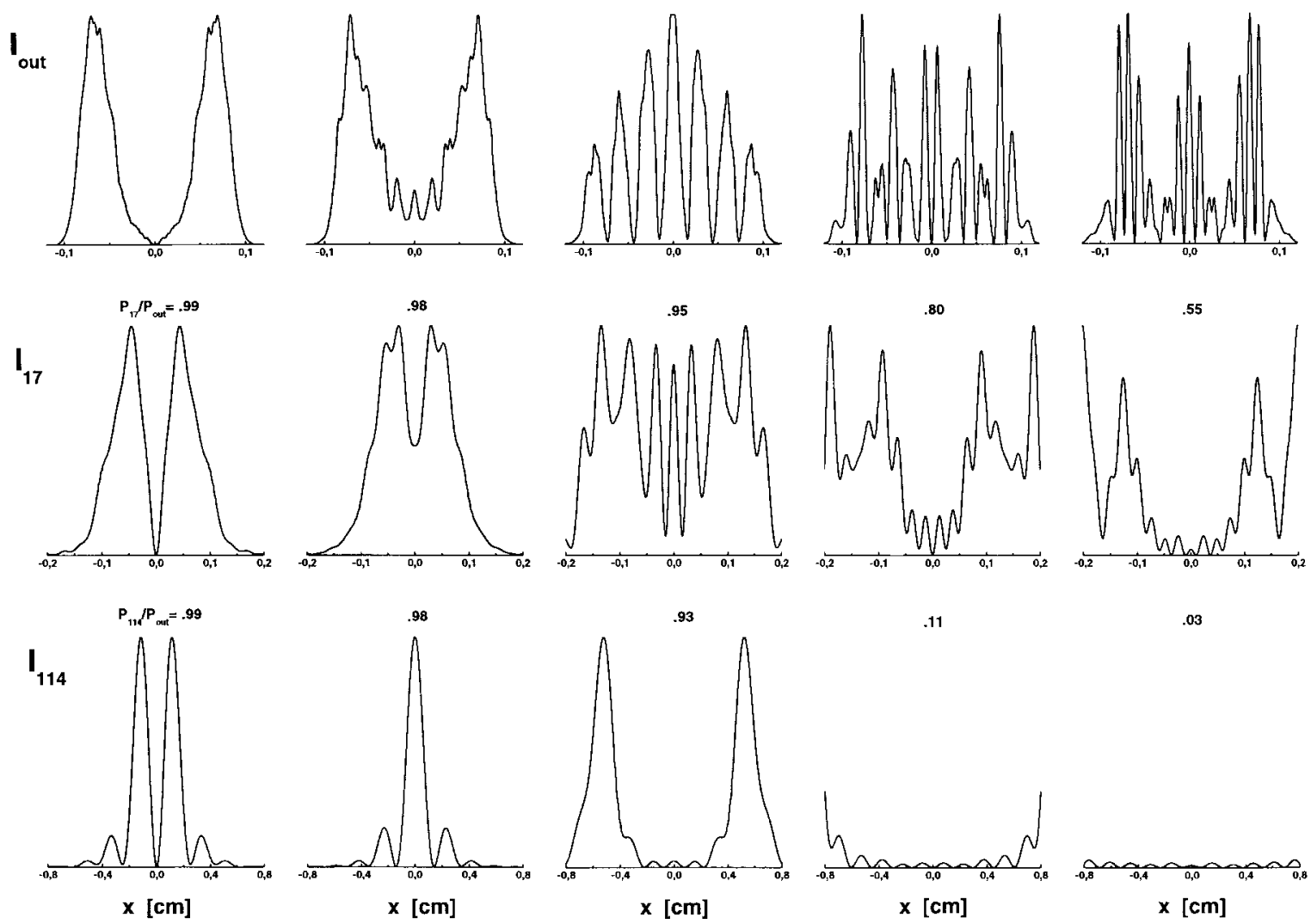

Fig. 8. Five mode patterns calculated at the output mirror plane $\left(I_{\text {out }}\right)$ and at distances of $17\left(I_{17}\right)$ and $114\left(I_{114}\right)$ cm behind the output mirror. $\mathrm{Xe}: \mathrm{Ar}: \mathrm{He}=0.5: 59.5: 40 ; \lambda=2.65 \mu \mathrm{m}$.

Physically, the allowable magnitude of the gain coefficient may be evaluated from the condition

$$
g_{0} \leq k \Delta n
$$

where $\Delta n$ is the refractive index difference between the center and waveguide wall. With this condition fulfilled, the mode pattern is insensitive to the appearance of gain and may be found by calculations of diffraction equations for the cavity with a passive waveguide. The form-factor $\alpha_{n}$ depends on the cavity mode number $m$ and on the gain profile. Its magnitude is governed by an overlap of mode intensity and gain distributions. From simple considerations of the radiation balance within the laser cavity, it is evident that the laser radiation power emitted by the active medium is equal to $\iint g(x, z) I_{n}(x, z) d x d z$, where $I_{n}(x, z)$ is the mode intensity and the integration is performed over the waveguide volume. From the other side, it may be expressed using the modal gain as $\alpha_{n} g_{0} \iint I_{n}(x, z) d x d z$. Taking into account that the mode pattern does not depend on gain, the factor $\alpha_{n}$ can be found for the given gain profile from the expression

$$
\alpha_{n}=\frac{\iint g(x, z) I_{n}(x, z) d x d z}{g_{0} \iint I_{n}(x, z) d x d z} .
$$

It is important to emphasize that the waveguide coupling losses $\delta_{n}$ and wall absorption losses are almost independent of the amplitude and shape of the gain distribution and can be
TABLE II

Eigenfrequencies of Five Transverse Modes with CoRresponding Modal Gain $g_{m}$, Waveguide Coupling Losses $\delta_{c}$, And Waveguide Wali Absorption $\delta_{w}$. Gas Mixture at 150-mbar Pressure (40\% He Content), AN InPut Power of $350 \mathrm{~W}$, ANd Laser WaVELENGTH $\lambda=2.65 \mu \mathrm{m}$

\begin{tabular}{c|c|c|c|c|c}
\hline $\mathrm{n}$ & 1 & 2 & 3 & 4 & 5 \\
\hline$\Omega, \mathrm{MHz}$ & 363 & 378 & -348 & 336 & 162 \\
\hline $\mathrm{g}_{\mathrm{n}}, \mathrm{m}^{-1}$ & 5.14 & 4.75 & 4.41 & 4.47 & 4.49 \\
\hline$\delta_{\mathrm{c},} \%$ & 2.14 & 1.92 & 8.15 & 11.17 & 8.76 \\
\hline$\delta_{\mathrm{w}}, \%$ & 0.48 & 0.69 & 3.34 & 9.72 & 14.8 \\
\hline
\end{tabular}

found from the solution of the diffraction model for a passive waveguide.

In general, calculations of the characteristics of a cavity that contains an active waveguide can be performed in two stages. First, for the given cavity configuration and refractive index distribution, the optical mode patterns are calculated and the following quantities are found: $\delta_{w}$ and $\delta_{c}$. At the second step, a simple integration is made according to (12) for the given gain profile. The modal gain is simply calculated from the linear relationship (10). The eigennumber can be calculated by simple algebra from (9).

Calculations showed that, for the $90 \%$-He mixture where the effect of refractive index gradients was remarkably weaker, transverse modes are discriminated against quite poorly. To illustrate this fact, the computed dependence of the eigennumbers for the lowest three even and three odd modes on the amplitude of the small-signal gain is displayed in Fig. 9. Calculations were made for the $90 \%-\mathrm{He}$ mixture for 


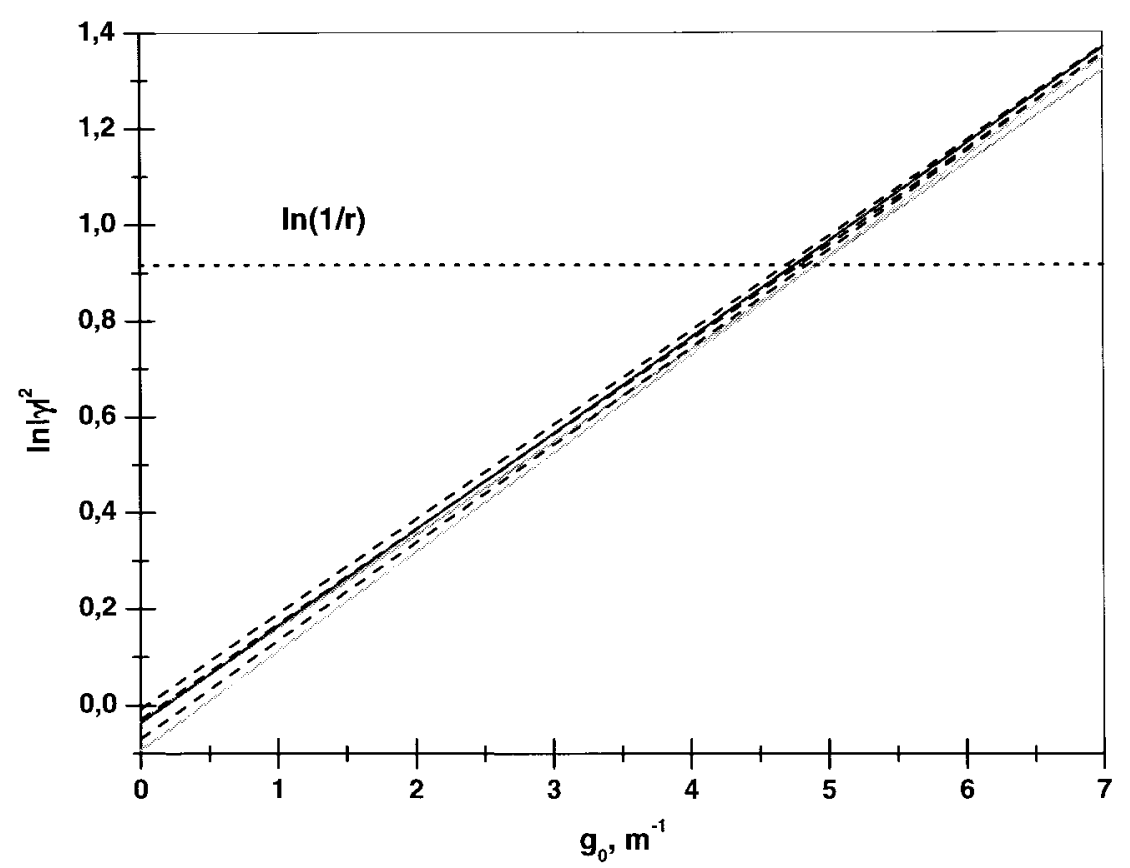

Fig. 9. Calculated dependence of eigennumber moduli for first three even (solid lines) and three odd (dashed lines) transverse modes. Xe:Ar:He $=$ $0.5: 9.5: 90 ; \lambda=2.03 \mu \mathrm{m}$.

$\lambda=2.03 \mu \mathrm{m}$ while the other conditions were the same as for the results presented in Fig. 5. It is seen in Fig. 9 that discrimination between modes for gain values in the vicinity of threshold is very weak. It is evident that, when the small-signal gain is above threshold, the effect of nonuniform saturation will result in simultaneous lasing of many cavity modes. This situation is completely different from that existing for the $40 \%$ He mixture. Fig. 9 shows that one may expect simultaneous lasing of six transverse modes, while from Fig. 5 it follows that most probably only two modes or their linear combination may be involved in lasing.

\section{COMPARISON WITH EXPERIMENTAL DATA}

Studies in the previous section highlighted the interplay of such factors as diffraction, nonuniform gain, and refractive index for conditions close to the experimental ones. The purpose of this section is to directly compare the output radiation patterns measured experimentally at two positions 17 and $114 \mathrm{~cm}$ from the output mirror with the calculated patterns. The procedure we took is based on the assumption that experimental intensity profiles can be represented as a modal intensity expansion with coefficients found from the least-mean-square calculations. Looking at the results of experimental measurements (Figs. 10-14, solid lines), some asymmetry of the distributions can be seen. Reasons for the appearance of this asymmetry are not identified. However, it is clear that an agreement between theory and experiment may be expected only when this asymmetry is neglected.

As a basis for the expansion of the far-field intensity distribution for the $90 \%$ He mixture, the first three even and three odd modes were calculated for conditions comparable to the experimental ones. The numerical coefficients were calculated by comparing the measured distribution with the

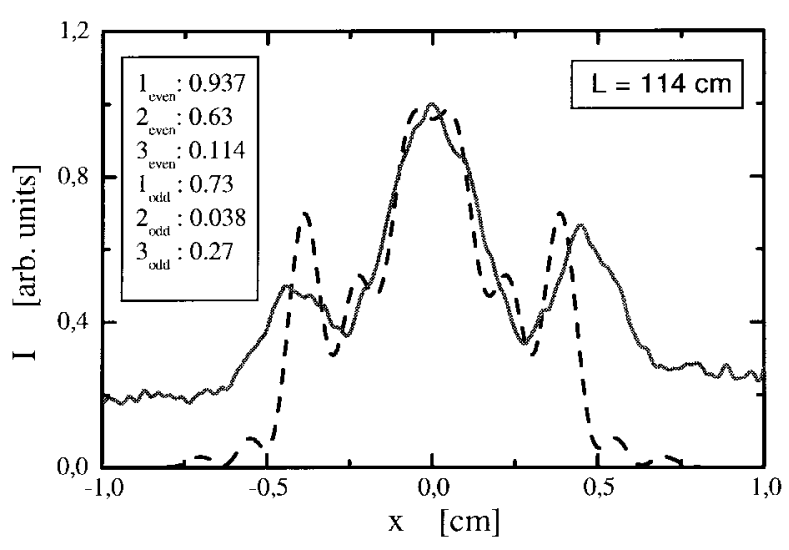

(a)

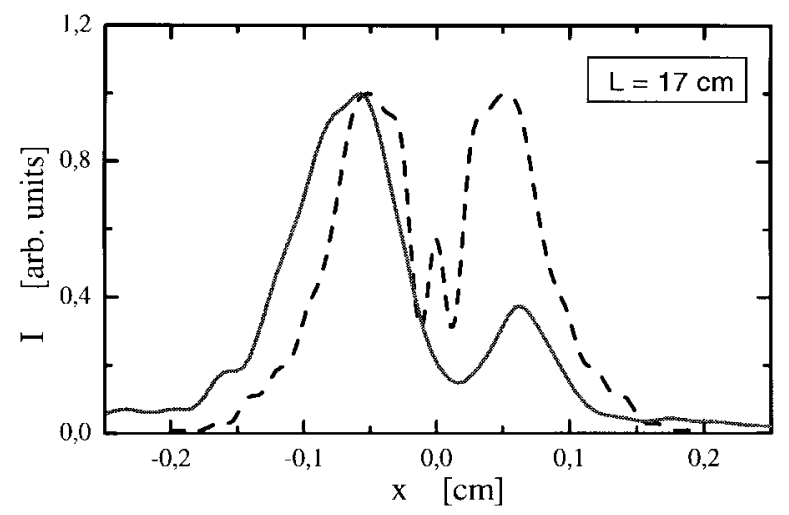

(b)

Fig. 10. Results of a least-square fit using three even and three odd mode intensities with (a) the experimental far-field pattern $(114 \mathrm{~cm})$ and (b) a comparison of the calculated and measured laser intensities at a distance 17 $\mathrm{cm}$ behind the output mirror. The fit coefficients are listed in the table placed in the figure. $(\mathrm{Xe}: \mathrm{Ar}: \mathrm{He}=0.5: 9.5: 90 ; \lambda=2.03 \mu \mathrm{m})$. 


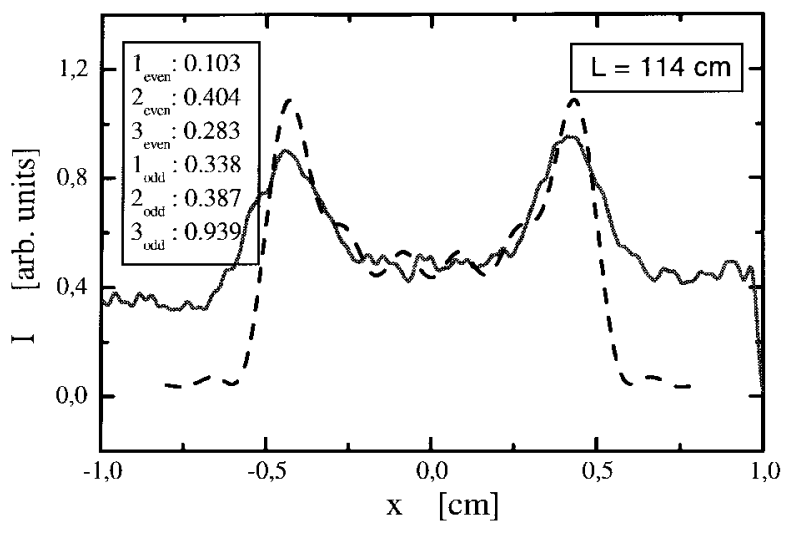

(a)

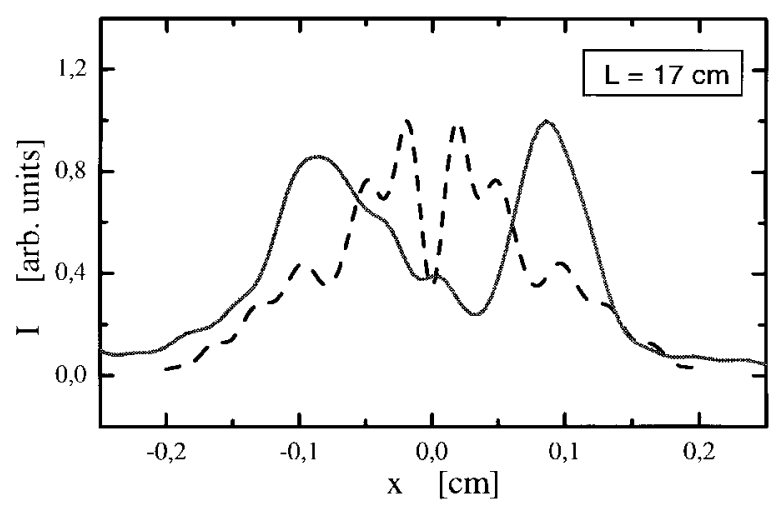

(b)

Fig. 11. Results of a least-square fit using three even and three odd mode intensities with (a) the experimental far-field pattern $(114 \mathrm{~cm})$ and (b) comparison of the calculated and measured laser intensities at distance 17 $\mathrm{cm}$ behind the output mirror. (Xe : $\mathrm{Ar}: \mathrm{He}=0.5: 9.5: 90 ; \lambda=2.65 \mu \mathrm{m}$ ).

sum of these six mode intensities and adjusting the coefficients to minimize the difference between both quantities. The results are presented in Figs. 10-12 for the 90\% He mixture and laser wavelengths of 2.03, 2.65 and $3.51 \mu \mathrm{m}$, respectively. The solid lines show the experimental data and the broken lines display the results of a best fit to the far-field $(114 \mathrm{~cm})$ distributions. Next, with the same coefficients, the distributions at a distance of $17 \mathrm{~cm}$ from the output mirror have been calculated. The magnitudes of the numerical coefficients found are listed in the figure captions. One can see that all the coefficients are positive which is important from the point of view of the physical meaning of these coefficients. Bearing in mind the poor pattern reproducibility in the experiments and the lack of expected symmetry, the agreement between theoretical and calculated curves may be considered satisfactory. It is also important to note that the calculated laser beam divergence turns out to be quite close to the experimental one. It is easy to determine that this divergence is approximately four, three, and two times greater than the corresponding diffraction limit for $2.03,2.65$ and $3.51 \mu \mathrm{m}$, respectively. Having no data about the spatial distribution of the gain, the same distribution was taken as in the calculations for the $40 \% \mathrm{He}$ mixture. Therefore, the results obtained can be considered as typical for an Ar-Xe laser with a He diluted laser gas mixture.

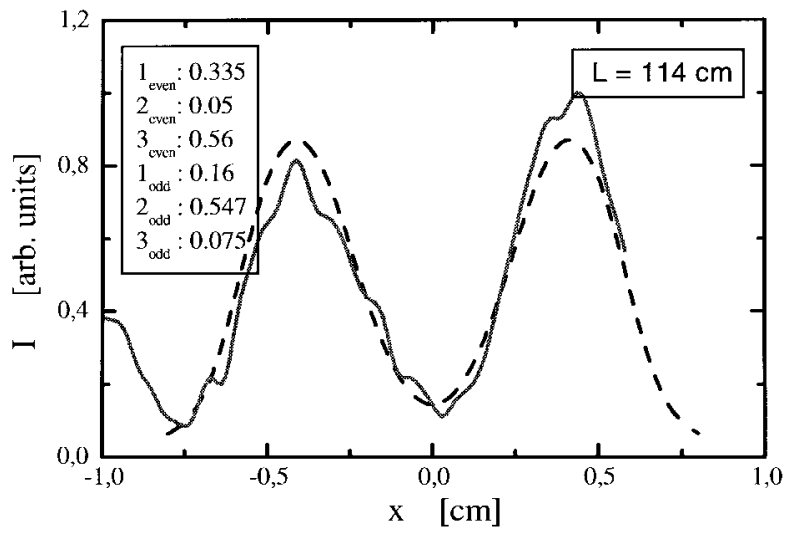

(a)

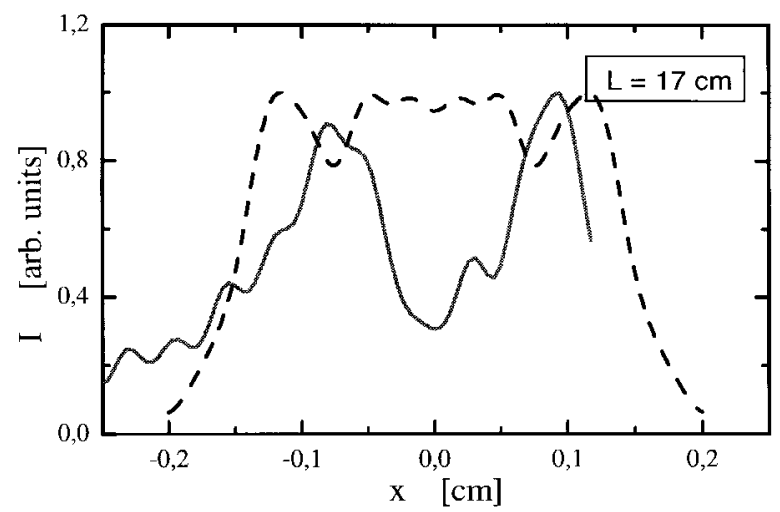

(b)

Fig. 12. Results of a least-square fit using three even and three odd mode intensities with (a) the experimental far-field pattern $(114 \mathrm{~cm})$ and (b) a comparison of the calculated and measured laser intensities at distance 17 $\mathrm{cm}$ behind the output mirror. (Xe: $\mathrm{Ar}: \mathrm{He}=0.5: 9.5: 90 ; \lambda=3.51 \mu \mathrm{m}$ ).

A similar procedure was used to generate the data for the $2.65-\mu \mathrm{m}$ laser radiation in a $40 \% \mathrm{He}$ mixture. The results are shown in Fig. 13. It is worth noting that, for these calculations, only three modes of those presented in Fig. 8 have to be used for the approximation of the experimental data. The expansion coefficients are also positive. For $\lambda=2.03 \mu \mathrm{m}$ in a $40 \% \mathrm{He}$ mixture, it is seen in Fig. 5 that the two first modes are far separated by the modal gain-loss from all higher order modes. In the near field $(17 \mathrm{~cm})$, these modes have almost identical intensity profiles and are not similar to the experimentally observed distribution with a single maximum (Fig. 14). The discharge excitation asymmetry or misalignment of the cavity elements can explain the lack of symmetry observed in the experimental patterns. We are able to simulate numerically these asymmetry effects by introducing variations in the gain or refractive index profiles. Having no exact information on the nature and magnitude of the asymmetry in the laser cavity, the assumed asymmetrical gain profile was somewhat arbitrary. However, the results of our calculations have a simple interpretation in terms of two weakly coupled waveguides, as discussed above. The perturbation of symmetry is strong enough to make lasing in these waveguides independent (or incoherent). The experimental near-field pattern with one peak is evidence for 


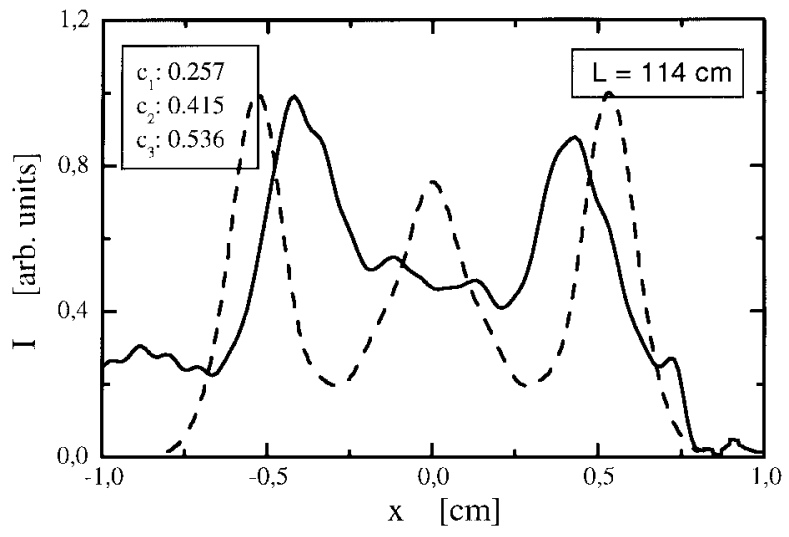

(a)

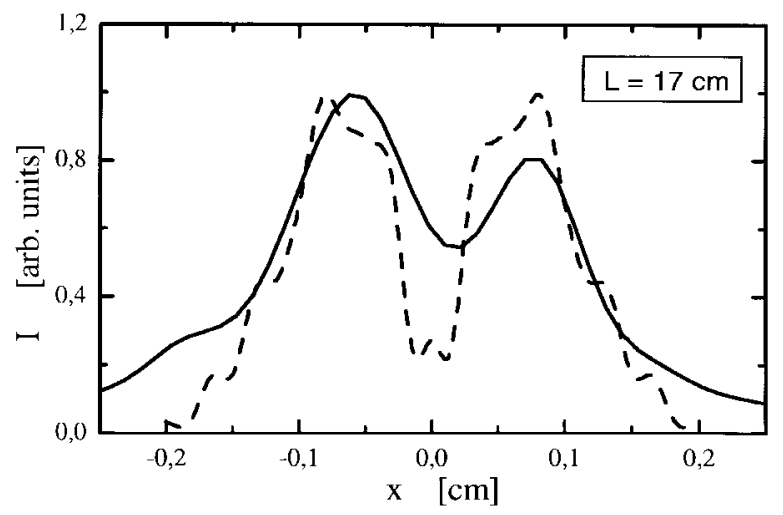

(b)

Fig. 13. Results of a least-square fit using the three lowest mode intensities with (a) the experimental far-field pattern $(114 \mathrm{~cm})$ and $(\mathrm{b})$ a comparison of the calculated and measured laser intensities at a distance $17 \mathrm{~cm}$ behind the output mirror. (Xe: $\mathrm{Ar}: \mathrm{He}=0.5: 59.5: 40 ; \lambda=2.65 \mu \mathrm{m}$ ).

a strong preference of the laser intensity in one of these waveguides. Therefore, Fig. 14 shows a comparison of the calculated dominant mode patterns at distances of 114 and 17 $\mathrm{cm}$, respectively, with experimental patterns obtained at the same distances. It is seen that the experimental distributions are about two times broader than the calculated ones. To get a better agreement between the experiment and theory, in this case, further efforts are necessary.

\section{CONCLUSIONS}

An optical diffraction model was developed for an $\mathrm{Ar}-\mathrm{He}-\mathrm{Xe}$ laser. It incorporates the effects of gain and refractive index gradients in the waveguide as well as diffraction in free space between the waveguide and the mirrors. The important role of all these factors was demonstrated for typical experimental conditions of an $\mathrm{Ar}-\mathrm{Xe}$ laser. The possibility of the formation of two weakly coupled effective waveguides in the vicinity of the dielectric waveguide walls was suggested. This effect is more pronounced in the case of Ar-rich mixtures. Studies were performed on the effect of a nonuniform gain distribution on the mode pattern. A simple procedure was developed to calculate the modal gain and losses. A direct comparison of the calculated mode patterns at different distances from the output mirror with experimentally

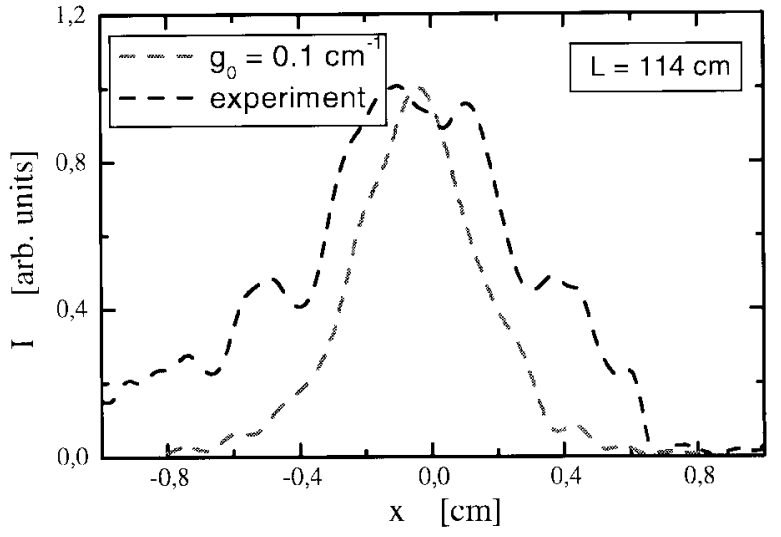

(a)

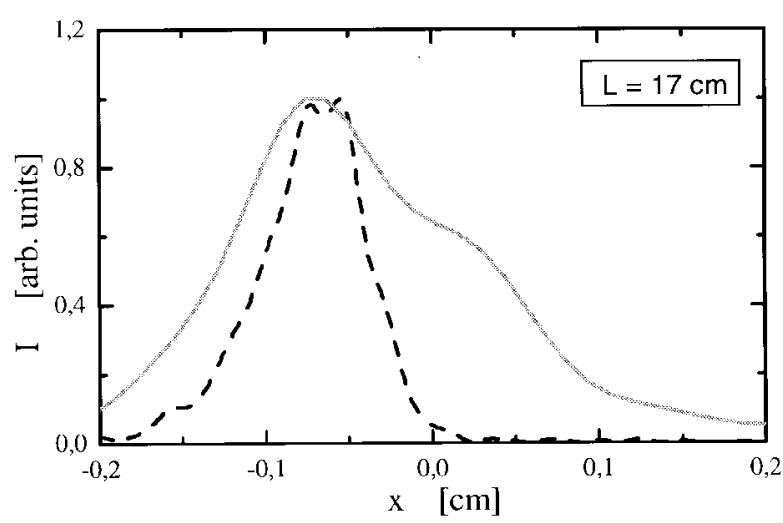

(b)

Fig. 14. Results of a least-square fit using one lowest mode intensity with (a) the experimental far-field pattern $(114 \mathrm{~cm})$ and (b) a comparison of the calculated and measured laser intensity at a distance $17 \mathrm{~cm}$ behind the output mirror. The assumed gain distribution was asymmetrical with respect to the waveguide center (shift of $0.03 \mathrm{~cm}$ ). (Xe: $\mathrm{Ar}: \mathrm{He}=0.5: 59.5: 40$; $\lambda=2.03 \mu \mathrm{m})$

measured patterns shows a satisfactory agreement for various laser wavelengths and gas mixtures.

\section{REFERENCES}

[1] Y. B. Udalov, P. J. M. Peters, M. B. Heeman-Ilieva, F. H. J. Ernst, V. N. Ochkin, and W. J. Witteman, "New continuous wave infrared Ar-Xe laser at intermediate gas pressures pumped by a transverse radio frequency discharge," Appl. Phys. Lett., vol. 63, pp. 721-722, 1993.

[2] S. N. Tskhai, Y. B. Udalov, P. J. M. Peters, W. J. Witteman, and V. N. Ochkin, "Continuous wave near-infrared atomic Xe laser excited by a radio frequency discharge in a slab geometry," Appl. Phys. Lett., vol. 66, pp. 801-803, 1995.

[3] P. P. Vitruk, R. J. Morley, H. J. Baker, and D. R. Hall, "High power continuous wave atomic Xe laser with radio frequency excitation," Appl. Phys. Lett., vol. 67, pp. 1366-1368, 1995.

[4] J. J. Wendland, R. J. Morley, H. J. Baker, and D. R. Hall, "High power mid infrared operation of the atomic xenon laser," Appl. Phys. Lett., vol. 72, pp. 1436-1438, 1998.

[5] S. N. Tskhai, F. J. Blok, Y. B. Udalov, P. J. M. Peters, W. J. Witteman, and V. N. Ochkin, "Spatial gain profiles of a continuous wave radiofrequency pumped atomic xenon laser," Appl. Phys. Lett., vol. 70, pp. 1921-1922, 1997.

[6] Y. P. Raizer, M. N. Schneider, and N. A. Yatsenko, Radio-Frequency Capacitive Discharges. Boca Raton, FL: CRC, 1995.

[7] B. Schroder, "Transverse modes of active hollow waveguide resonators," IEEE J. Quantum Electron., vol. 27, pp. 158-165, 1991. 
[8] F. J. Blok, I. V. Kochetov, A. P. Napartovich, V. N. Ochkin, P. J. M. Peters, S. A. Starostin, Y. B. Udalov, and W. J. Witteman, "The spatial structure of a transverse RF excited $\mathrm{Ar}-\mathrm{He}-\mathrm{Xe}$ laser," presented at the 1997 IEEE/LEOS Symp., Eindhoven, The Netherlands, 1997.

[9] J. A. V. Eijkelenhorg, A. M. Lindberg, M. S. Thijssen, and J. P. Woerdman, "Higher order transverse modes of an unstable-cavity laser," IEEE J. Quantum Electron., vol. 34, pp. 955-965, 1998.

[10] S. N. Tskhai, Y. B. Udalov, P. J. M. Peters, W. J. Witteman, and V. N. Ochkin, "Spectral investigation of cw rf-pumped atomic Xe laser with a slab geometry," Appl. Phys., vol. 62, pp. 11-14, 1996.

[11] M. J. Adams, Introduction to Optical Waveguides. Chichester, U.K.: Wiley, 1981.

[12] D. K. Faddeev and V. N. Faddeeva, Computational Methods of Linear Algebra. San Francisco, CA: Freeman, 1963.

[13] R. L. Abrams and A. N. Chester, "Resonator theory for hollow waveguide lasers," Appl. Opt., vol. 13, pp. 2117-2125, 1974.

[14] R. Gerlach, D. Wei, and N. M. Amer, "Coupling efficiency of waveguide laser resonators formed by flat mirrors: Analysis and experiment," IEEE J. Quantum Electron., vol. QE-20, pp. 948-963, 1984.

[15] J. Henningsen, M. Hammerich, and A. Olaffson, "Mode structure of hollow dielectric waveguide lasers," Appl. Phys. B, vol. 51, pp. 272-284, 1990.

Frans J. Blok was born on November 10, 1969. He received the degree in physics in May 1995. His thesis was on the quasi-static structure of atomic valence electrons during collisions, performed in the Quantum Optics Group at Leiden University, The Netherlands. He is currently working towards the $\mathrm{PhD}$. degree in the Laser Physics group at the University of Twente, Enschede, The Netherlands, investigating RF excited atomic xenon lasers.

Nikolay N. Elkin was born in Kirov province, Russia, on December 10 , 1951. He graduated from Moscow State University in 1976 and received the Kandidat degree in theoretical and mathematical physics and the Doctor of Science degree in mathematical modeling in science from Moscow State University in 1980 and 1998, respectively. His Kandidat dissertation was devoted to the boundary problems of the tidal waves theory.

In 1979, he joined Branch of Kurchatov Atomic Energy Institute. After the kandidat dissertation defense, his research concentrated on the numerical modeling of gas discharge and then on the mathematical modeling in nonlinear and resonator optics for fast-flow $\mathrm{CO}_{2}$ lasers. From 1979 to 1987, from 1987 to 1988 , and from 1988 to 1994 , he was Junior Researcher, Researcher, and Senior Researcher, respectively, with the Branch of Kurchatov Institute. Under his active participation, the comprehensive computer models for $\mathrm{CO}_{3}$ and CO lasers were created and succesfully used for theoretical description of this laser. Since 1994, he is a leading researcher with the Troitsk Institute for Innovation and Fusion Research (renamed from Branch of Kurchatov Institute). He has over 50 technical publications. Recently, his interests shifted to mathematical modeling in the theory of lasers with diffractive optical elements, waveguide lasers, and nonstationary lasing. Since 1994, he has also delivered lectures in computational physics at the Moscow Institute of Physics and Technology.

Dr. Elkin received the Kurchatov Award in 1986. He is a member of SPIE.
Igor V. Kochetov was born in Kursk, Russia, on January 23, 1951. He graduated from the Department of Radiotechnics and Cybernetics of the Moscow Institute of Physics and Technology (MIPT) in 1974 and received the Kandidat of Science degrees in radio physics from MIPT in 1977. His kandidat dissertation was devoted to the numerical simulation of physical processes in discharge pumped CO lasers.

In 1978, he joined the Special Design Bureau "Almaz," Moscow. In 1981, he joined Branch of Kurchatov Atomic Energy Institute, Troitsk, Moscow region. After the kandidat dissertation defense, his research concentrated mainly on the theory of a gas discharge and gas lasers. From 1978 to 1993, he held the position of Senior Researcher, and in 1993 he became Leading Researcher. His research focused on the development of theoretical and numerical modeling of electrons, ions, molecular vibration, and chemical kinetics in nonequilibrium plasmas, modeling of gas lasers, low-temperature plasma, and pulsed, dc, and RF discharges. He has contributed to theory of the vibrational kinetics, calculations of the electron rate coefficients in gases under nonuniform nonstationary field and electron density conditions, the theory of the negative differential conductivity under different conditions, the kinetics of the $\mathrm{CO}_{2}$ and $\mathrm{CO}$ lasers, the kinetics of excimer and ionic excimer laser, and ionization plasma instabilities and spatio-temporal evolution of gas discharge during constriction of its plasma column. Currently, he is performing simulation of corona discharge and modeling the waveguide laser with RF pumping. He has authored over 200 technical publications.

Dr. Kochetov received the Award of the Government of the Russian Federation.

Anatoly P. Napartovich was born in Ulan-Ude, Russia, on December 1, 1940 He graduated from the Moscow Institute of Physics and Technology in 1964 and received the Kandidat and Doctor of Science degrees in plasma physics from the High-Temperature Institute (Moscow) in 1969 and from Kurchatov Atomic Energy Institute in 1980, respectively. His kandidat dissertation was devoted to the resonance radiation transfer theory.

In 1967, he joined the Kurchatov Atomic Energy Institute. After the kandidat dissertation defense, his research concentrated mainly on the theory of a gas discharge and resonator optics for fast-flow $\mathrm{CO}_{2}$ lasers. From 1967 to 1972 and from 1972 to 1982 , he was a Junior Researcher and Senior Researcher, respectively, with the Branch of Kurchatov Institute. He predicted several kinds of plasma and optical instabilities limiting the power and optical quality of laser beams. Under his guidance, the comprehensive computer models for $\mathrm{CO}_{2}, \mathrm{CO}$, and excimer lasers were formulated and successfully used for the theoretical description of these lasers. Since 1982, he has been a head of the Gas Laser Theory Lab. Recently, his interests shifted to the theory of optically coupled laser arrays. He proposed a new type of coupling based on the Talbot effect, which was realized in an experiment. He is also involved in the work on the glow discharge in an air flow at atmospheric pressure, which is very promising for applications in flue-gas cleanup. He has over 300 technical publications and holds six inventions. Since 1973, he also delivers lectures in laser physics for students of Moscow Institute of Physics and Technology. Since 1984, he held a half-time position of Professor at this Institute.

Peter J. M. Peters was born in Meerlo, The Netherlands, on November 5, 1945. He received the M.Sc. degree from the Catholic University, Nijmegen, The Netherlands and the Ph.D. degree in laser physics from the University of Twente in 1981

He then joined the staff of the Quantum Electronics Group of the Department of Applied Physics of the University of Twente, where he is now an Associate Professor. He has carried out research mainly in the field of gas lasers. His Ph.D. work on a pulsed TEA CO laser was followed by research work on different types of excimer lasers like $\mathrm{KrF}, \mathrm{ArF}, \mathrm{XeF}(\mathrm{CA}$ and $\mathrm{BX}$ ) and on ionic excimers. Currently, he is engaged in research on the VUV molecular F2 laser and in the rare gas recombination lasers like the atomic Xe laser emitting in the near infrared. 
Sergei A. Starostin was born on August 7, 1970, in Moscow, Russia. He graduated from the Departement of Plasma Physics, Moscow Engeneering Physics Institute, Moscow, in 1993. He is currently working towards the Ph.D. degree at University of Twente, Enschede, The Netherlands.

From 1993 to 1996, he worked as an Engineer-Reseacher at the Lebedev Physical Institute, Moscow. His current research interests are in the fields of gas discharge lasers, numerical modeling of gas discharges, and physics of laser resonators.

Vera N. Troshchieva was born in Grodno, Belorussia, May 24, 1963. She graduated from Department of Computational Mathematics and Cybernetics of Moscow State University in 1985.

Since then, she has been an Engineer woith Troitsk Institute for Innovation and Fusion Research, Troitsk, Moscow region. She is engaged in numerical diffraction simulations of optics of composite laser systems (a laser controlled by injection of an external signal, a waveguide laser with RF pumping, etc.). She has over ten technical publications.

Yuri B. Udalov was born in Yuzhno-Sakhalinsk, Russia, on January 29, 1955. He graduated cum laude from Moscow Engineering Physics Institute (MEPhI) in 1978. He received the Ph.D. degree in radio physics from the P.N. Lebedev Physics Institute, Moscow, Russia, in 1984.

In 1978, he began to work in the Laboratory of Low Temperature Plasma Optics of the P.N. Lebedev Physics Institute as an Engineer, Junior Research Associate, and later on as Research Associate. Since 1990, he has worked as a Post-Doctoral Associate in the group of Quantum Electronics at the University of Twente, The Netherlands. During his research career, he was involved in the research on laser resonators, frequency selection and stabilization, studies on $\mathrm{CO}_{2}$ and $\mathrm{CO}$ electric discharge gas lasers, techniques for gas discharge stabilization, gas discharge assisted catalysis, and spectroscopy of low temperature plasmas. He is now actively engaged in the field of RF excitation of gas lasers systems, especially neutral xenon and $\mathrm{CO}_{2}$ lasers. His recent research interests also include gas discharge physics, problems of discharge stability, lidars, and remote sensing. He has authored approximately 80 scientific publications.
W. J. Witteman was born in Monster, The Netherlands, on December 12, 1933. He received a degree of mechanical engineer from the Technical University of Delft, The Netherlands, in 1958 and the Ph.D. degree in physics from the Technical University of Eindhoven, Eindhoven, The Netherlands, in 1963.

From 1958 to 1960 , he was first a Post-Doctoral Fellow and later a Research Associate at the Institute of Fluid Dynamics and Applied Mathematics, University of Maryland, College Park, studying molecular relaxation phenomena by means of shock tube experiments. From 1961 until 1969, he was with Philips Research Laboratories, Eindhoven, The Netherlands, where he was engaged with high-pressure physics and technology until 1963. After that, he performed research on water vapor lasers, sealed-off $\mathrm{CO}_{2}$ lasers, and argon-ion lasers. Since 1969, he has been a professor at the University of Twente, Enschede, The Netherlands, where he is working on high-power pulsed laser systems. He is actively engaged in the field of $\mathrm{CO}_{2}$ lasers, mode-locking techniques, electron beam and discharge pulsed excimer lasers, both continuous and pulsed CO-lasers, electro-ionisation lasers like $\mathrm{Ar}-\mathrm{Xe}$, waveguide lasers, and free electron lasers of the Raman and Compton type. In 1989, he also became Managing Director of Nederland Centrum voor Laser Research (NCLR) B.V., which is a joint venture between the University of Twente and the industry. The NCLR developes advanced laser systems for industrial applications like a 1-kW Eureka-excimer laser operating at $1 \mathrm{kHz}$. He retired from both positions in 1998 